\title{
Association of tumor size and internal echo pattern with coagulopathy associated with hepatic hemangioma
}

\author{
SHIGEO MARUYAMA ${ }^{1}$, MASAHIKO KODA ${ }^{2}$, TOMOMITSU MATONO ${ }^{3}$ and HAJIME ISOMOTO ${ }^{3}$ \\ ${ }^{1}$ Department of Internal Medicine, Maruyama Medical Clinic, Hamada, Shimane 697-0034; ${ }^{2}$ Department of Internal Medicine, \\ Hino Hospital, Hino, Tottori 689-4504; ${ }^{3}$ Department of Multidisciplinary Internal Medicine, Faculty of Medicine, \\ Tottori University, Yonago, Tottori 683-8504, Japan
}

Received June 30, 2020; Accepted February 5, 2021

DOI: $10.3892 / \operatorname{mco} .2021 .2245$

\begin{abstract}
Little is known concerning causal factors associated with the size and echogenicity of hepatic hemangiomas. The aim of the present study was to investigate the associations between tumor size and echo pattern and coagulation factors, and to elucidate the growth pattern of hemangiomas. In 214 consecutive patients with hepatic hemangiomas, ultrasonography was performed to determine total tumor number, size, echogenicity and location, and serum laboratory tests for liver function and coagulation factors were carried out. The ultrasonographic appearance of hemangiomas was homogeneous in $75.7 \%$ of cases and mixed in $24.3 \%$ of cases. A mixed echo pattern was seen in 1 out of 145 masses $(0.7 \%)$ with a diameter $<20 \mathrm{~mm}$, in 30 out of $48(62.5 \%)$ with a diameter of $20-40 \mathrm{~mm}$, and in all of the $21(100 \%)$ with a diameter $>40 \mathrm{~mm}$. Platelet counts $(\mathrm{P}<0.0001)$ and fibrinogen levels $(\mathrm{P}<0.01)$ were lower in patients with larger and mixed tumors. Levels of thrombin-antithrombin III complex (TAT), D-dimer, and fibrin and fibrinogen degradation products (FDP) were significantly elevated along with an increase in tumor size (all $\mathrm{P}<0.0001)$, and the number of patients with the abnormal values of TAT, D-dimer, and FDP was significantly higher in the mixed group than in the homogeneous group (all $\mathrm{P}<0.0001)$. Fibrinogen $(\mathrm{P}<0.01)$, platelet count $(\mathrm{P}<0.001)$, portal vein diameter $(\mathrm{P}<0.0001)$, splenic index $(\mathrm{P}<0.01)$, and levels of TAT, $\mathrm{D}$-dimer and FDP (all $\mathrm{P}<0.0001)$ were significantly associated with tumor
\end{abstract}

Correspondence to: Dr Masahiko Koda, Department of Internal Medicine, Hino Hospital, Nota 332, Hino, Tottori 689-4504, Japan E-mail: masakoda89@yahoo.co.jp

Abbreviations: ALT, alanine transeferase; GGT, $\gamma$-glutamyltranspeptidase; ALP, alkaline phosphatase; BUN, blood urea nitrogen; $\mathrm{Cr}$, creatinine; LDL, low density lipoprotein; WBC, white blood cell; AFP, $\alpha$-fetoprotein; PT, prothrombin time; TAT, thrombin-antithrombin III complex; FDP, fibrin and fibrinogen degradation products; US, ultrasonography; CT, computed tomography; PIVKA-II, protein induced by vitamin $\mathrm{K}$ absence-II; M2BPGi, Mac-2 binding protein glycosylation isomer; KMS, Kasabach-Merritt syndrome

Key words: hemangiomas, coagulation factors, thrombosis size. Multivariate analysis revealed TAT, D-dimer and FDP as independent predictors of tumor size. The internal echo pattern became mixed as size increased. The size and echogenicity of hemangiomas were closely associated with coagulation factors. Therefore, it was speculated that differences in size and echogenicity were caused by intratumoral thrombosis and subsequent hemorrhage.

\section{Introduction}

Hepatic hemangioma is the most common benign tumor of the liver, with a prevalence of $0.4-7.3 \%$, and an incidence of $1.7 \%$ on abdominal ultrasonographic examination $(1,2)$. Hepatic hemangiomas are composed of multiple and large vessels lined by a single layer of endothelial cells within a thin fibrous stroma $(2,3)$. Advances in abdominal imaging technology and their widespread application have improved the detection of benign liver tumors and most hepatic hemangiomas can be accurately diagnosed by imaging techniques. Ultrasonography (US) is the most commonly performed screening method because it is non-invasive, inexpensive, and commonly available, and has high specificity and sensitivity (3). On abdominal US, hemangiomas are generally visualized as homogeneous, hyperechoic, and well-defined lesions, although several studies have reported variations of these appearances in large lesions, and that the internal echoes become inhomogeneous as the mass increases in size $(2,4-7)$. However, little is known regarding the causes for the relationship between hemangioma size and echogenicity. While, it is well known that a giant hemangioma leads to coagulation abnormality, Kasabach-Merritt syndrome (KMS). However, the relationship between coagulation abnormality and hemangioma size or echogenicity is not clear. In most cases, hepatic hemangiomas are small and cause no symptoms. Therefore, most hepatic hemangiomas are diagnosed incidentally and have little clinical significance. Large hemangiomas occasionally can cause signs and symptoms sufficiently severe to require treatment. However, the management of patients with asymptomatic hemangiomas remains under debate. The reason is that previous studies have not shown the sufficient data that are available on the natural history of hepatic hemangiomas and their tendency to cause hemangioma-related complications. The aim of this study is to investigate the correlation 
between tumor size and the internal echo pattern revealed by US and the clinical factors, especially coagulation factors, to elucidate the natural history and growth pattern of hemangiomas.

\section{Patients and methods}

Patients. This study was approved by the ethics review board of Tottori university hospital (approval numbers: 18A023) and the ethical committee of Hino hospital (approval numbers: 2018-4). Of 16,403 abdominal US examinations performed at our hospitals between January 2016 and December 2018, 214 patients were diagnosed with hepatic hemangioma and were consecutively enrolled in this study after giving informed consent. Those with infectious diseases, liver cirrhosis, or malignant tumors were excluded from the study. Patients with abnormal values of thrombin-antithrombin III complex (TAT) $(>3.0 \mathrm{ng} / \mathrm{ml})$, D-dimer $(>1.0 \mu \mathrm{g} / \mathrm{ml})$, and fibrin and fibrinogen degradation products (FDP) $(>5.0 \mu \mathrm{g} / \mathrm{ml})$ were referred for cardiovascular medicine consultation to prevent complications of thrombosis such as deep vein thrombosis or lung thrombosis.

Methods. Hepatic hemangiomas were diagnosed by US and multiphase contrast-enhanced helical computed tomography $(\mathrm{CT})$. Their appearance on US is of homogeneous, hyperechoic, well-defined lesions, although larger hemangiomas showed mixed echogenicity. On CT, they were characterized as well-defined, homogeneous, hypodense lesions with peripheral nodular enhancement followed by progressive centripetal enhancement $(1,7)$. In the most hemangiomas (diameter $\leq 20 \mathrm{~mm}$ ), the final diagnosis was established based on the US features, clinical observation, and negative biochemical results. Relatively large lesions (diameter $>20 \mathrm{~mm}$ ) were diagnosed using both US and CT. Abdominal US was performed in all patients, and CT was performed in 67 patients. The US findings were reviewed and the following were recorded: Total number of hepatic hemangiomas, size, echogenicity, location, diameter of the portal vein, and the splenic index. In the case of multiple hemangiomas, the tumor with the largest diameter was selected for analysis. All patients were examined by US examiners who had 30 years of experience.

Hepatic hemangiomas can be divided into two major groups: Capillary hemangiomas and cavernous hemangiomas (2). In the present study, however, hemangiomas were not classified into the two tumor types due to difficulties in distinguishing them by US and CT. According to a previous study, most hemangiomas $(\sim 80 \%)$ are cavernous type (8). Using the method described in a previous study of giant cavernous hemangiomas (9), we divided the hepatic hemangiomas into three groups according to maximum diameter: Small group ( $<20 \mathrm{~mm})$, medium group $(20-40 \mathrm{~mm})$, and large group ( $>40 \mathrm{~mm})$. The internal echo pattern was classified into two groups: Homogeneous group (homogeneous hyperechoic, homogeneous hypoechoic, and isoechoic) and mixed group (mixed hyperechoic and mixed hypoechoic) (1).

Routine laboratory tests were performed using automated methods. Blood platelet counts in the range $14-38 \times 10^{4} / \mathrm{mm}^{3}$ were considered normal. Prothrombin time (PT) was determined by the coagulation method using one-stage prothrombin-time assay (Japan Clinical Laboratory, Kyoto, Japan), with values in the range 70-130\% considered normal. Fibrinogen was determined by rapid physiological coagulation technique using the clotting method (Japan Clinical Laboratory, Kyoto, Japan), with values in the range $180-400 \mathrm{mg} / \mathrm{dl}$ considered normal. Plasma TAT concentration was determined by a chemiluminescent enzyme immunoassay (Special Reference Laboratory, Tokyo, Japan). The normal TAT level is $<3.0 \mathrm{ng} / \mathrm{ml}$ and the lower detection limit is $1.0 \mathrm{ng} / \mathrm{ml}$. In 55 of 214 patients, the TAT level was $<1.0 \mathrm{ng} / \mathrm{ml}$. The concentrations of D-dimer and FDP were determined by a latex immunoturbidimetric assay (Japan Clinical Laboratory, Kyoto, Japan). The normal D-dimer and FDP levels are $<1.0$ and $<5.0 \mu \mathrm{g} / \mathrm{ml}$, respectively, with lower limits of sensitivity of $0.5 \mu \mathrm{g} / \mathrm{ml}$ for D-dimer and $2.6 \mu \mathrm{g} / \mathrm{ml}$ for FDP. In 77 and 187 of 214 patients, the D-dimer and FDP levels were $<0.5$ and $<2.6 \mu \mathrm{g} / \mathrm{ml}$, respectively. The values of TAT, D-dimer and FDP in 55, 77 and 187 of 214 patients were undetectable, therefore we categorized measured values as normal (TAT $<3.0 \mathrm{ng} / \mathrm{ml}$, D-dimer $<1.0 \mu \mathrm{g} / \mathrm{ml}, \mathrm{FDP}<5.0 \mu \mathrm{g} / \mathrm{ml})$ and abnormal value $(\mathrm{TAT} \geq 3.0 \mathrm{ng} / \mathrm{ml}, \mathrm{D}$-dimer $\geq 1.0 \mu \mathrm{g} / \mathrm{ml}, \mathrm{FDP} \geq 5.0 \mu \mathrm{g} / \mathrm{ml})$ and analyzed the difference among groups.

Follow-up study was performed with repeated US and blood examinations in patients with follow-up period over at least one year. The largest diameter of each tumor was recorded on the printed US images. Of the 214 patients with hepatic hemangiomas, 93 patients received at least two US and coagulation examinations in the follow-up period. The average follow-up period after the initial examination was 21.1 months (range, 12-29 months).

Statistical analysis. All measurements are expressed as the mean \pm SD. Differences among the three size groups were analyzed using one-way analysis of variance and Tukey's post hoc test, and the differences between the two echo pattern groups were analyzed using an unpaired t-test. Categorical variables were analyzed using the Chi-square test. Correlation analysis was carried out by univariate linear regression analysis. The multivariate forward stepwise regression analysis was used to identify independent factors related to the tumor size of hepatic hemangiomas. The data were analyzed using Stat Flex version 6.0 (Artech Co., Ltd.). A P-value $<0.05$ was considered statistically significant.

\section{Results}

Patient characteristics. Fig. 1 shows the internal echo patterns of hemangiomas according to mean tumor diameter. Most of small group were homogeneous. As the size increased, the internal echo pattern became the mixed echo type.

Among the 216 patients with hepatic hemangiomas, 2 patients were excluded because of high values of protein induced by vitamin K absence-II (PIVKA-II), which prevented differentiation from malignant tumors. A total of 214 patients were enrolled in the present study. The laboratory findings of these patients are listed in Table I. There were 74 men and 140 women (male:female ratio, 1:1.9), with a median age of 55 years (range, 23-89 years). All patients, except one patient with abdominal distension, were asymptomatic, and their hemangiomas had been discovered at a routine health exami- 


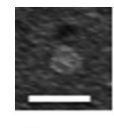

$5 \mathrm{~mm}$

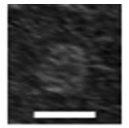

$10 \mathrm{~mm}$

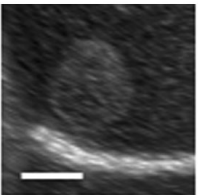

$20 \mathrm{~mm}$

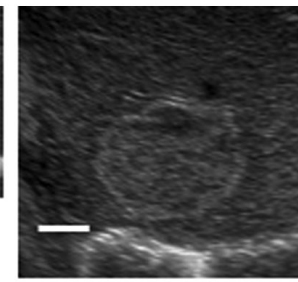

$30 \mathrm{~mm}$

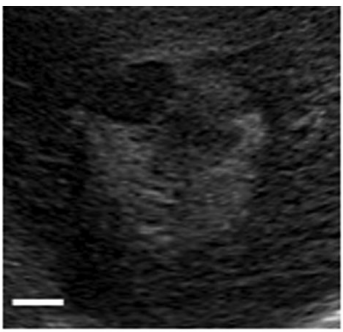

$40 \mathrm{~mm}$

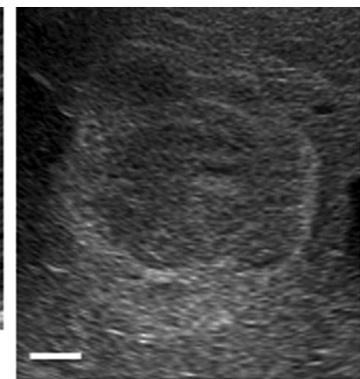

$50 \mathrm{~mm}$

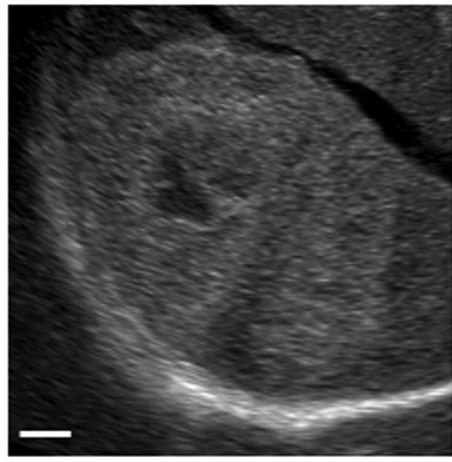

$80 \mathrm{~mm}$

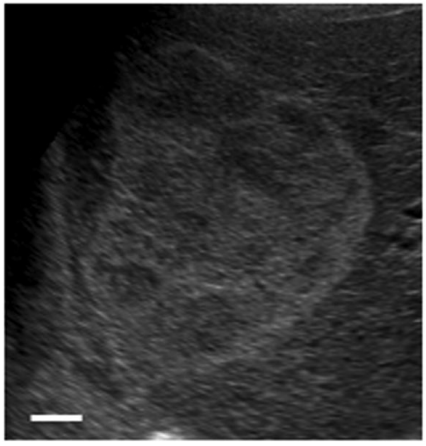

$70 \mathrm{~mm}$

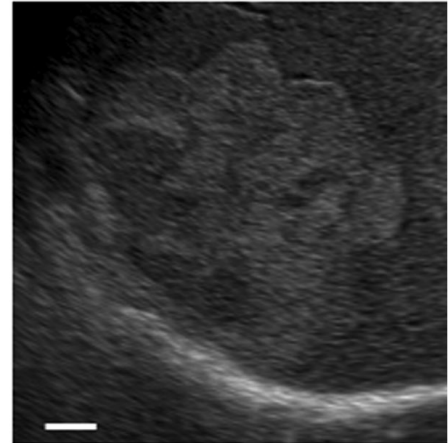

$60 \mathrm{~mm}$

Figure 1. Internal echo pattern according to different size of hemangiomas. Scale bars, $10 \mathrm{~mm}$.

nation or as an incidental finding on radiological studies. At the time of diagnosis, serum laboratory tests were normal in 190 of $214(88.8 \%)$ patients. Twenty-four patients had slight elevation in transaminases, glutamyl transpeptidase, or alkaline phosphatase. Thirty-two patients $(15.0 \%)$ had underlying chronic liver disease. No patient had received surgical treatment.

The US findings are listed in Table II. The sonographic appearance of the hemangiomas was hyperechoic in 159 $(74.3 \%)$, hypoechoic in $2(0.9 \%)$, and isoechoic in $1(0.5 \%)$ patient. The lesions presented a mixed echo pattern in $52(24.3 \%)$, with hyperechoic areas alternating with hypoechoic or anechoic areas. $145(67.8 \%)$ patients were in small group, $48(22.4 \%)$ in medium group, and $21(9.8 \%)$ in large group. The lesions were located in the right liver lobe in $180(84.1 \%)$, in the left liver lobe in $32(15.0 \%)$, and bilaterally in $2(0.9 \%)$ patients. A single hemangioma was detected in $173(80.8 \%)$ patients. The remaining $41(19.2 \%)$ patients had multiple tumors. Median portal diameter and splenic index were $10.8 \mathrm{~mm}$ and $1,295 \mathrm{~mm}^{2}$, respectively.

Association among hemangioma size, echo patterns and clinical parameters including coagulation factors. Table III shows the relationship of tumor size to the clinical parameters. There was no significant difference in terms of mean age or the male:female ratio among the three groups. Albumin concentration was significantly lower in the large group than in the other groups $(\mathrm{P}<0.01)$, but no biochemistry values except albumin and BUN showed significant differences among the three groups. Hemoglobin concentration was significantly lower in the large group than in the medium group $(\mathrm{P}<0.05)$. Values of Mac-2 binding protein glycosylation isomer (M2BPGi) were significantly higher in the large group than in the small group $(\mathrm{P}<0.05)$ and PIVKA-II levels were significantly higher in the medium and large group (both $\mathrm{P}<0.01$ ) than in the small group. Almost all tumors $(99.3 \%)$ in the small group were homogenous. In the medium group, a homogeneous pattern was seen in $37.5 \%(18 / 48)$ of hemangiomas and a mixed echo pattern in $62.5 \%$ (30/48). All hemangiomas in the large group had mixed echo patterns. Portal vein diameter was significantly higher in the medium and large group (both $\mathrm{P}<0.01$ ) than in the small group. Table IV shows the relationship of the echo pattern to the clinical parameters. The mean age and the male:female ratio were similar in the homogeneous and mixed groups. Albumin concentration was significantly lower $(\mathrm{P}<0.001)$ and the values of BUN, Cr, glucose, and PIVKA-II were significantly higher in the mixed group than in the homogeneous group. Tumor size in the mixed group was significantly larger than in the homogeneous group $(\mathrm{P}<0.0001)$. Portal vein diameter and splenic index were significantly elevated in the mixed group $(\mathrm{P}<0.001$ and $\mathrm{P}<0.05$, respectively).

Fig. 2 shows the relationship of tumor size to the coagulation markers (platelet, PT and fibrinogen). Platelet count was significantly lower in the large group than in the small and medium groups $(\mathrm{P}<0.01)$ and fibrinogen level was significantly lower in the large group than in the small group $(\mathrm{P}<0.01)$. PT was similar among all groups. Table V shows the relationship of tumor size to the coagulation markers (TAT, D-dimer and FDP). The number of patients with the abnormal value of TAT, D-dimer, and FDP significantly increased along with the tumor size (all $\mathrm{P}<0.0001$ ).

Fig. 3 shows the relationship of echo pattern to the coagulation markers (platelet, PT and fibrinogen). Platelet count $(\mathrm{P}<0.0001)$ and fibrinogen level $(\mathrm{P}<0.01)$ were significantly lower in the mixed group than in the homogeneous group. PT was similar in these two groups. Table VI shows the relationship of echo pattern to the coagulation markers (TAT, D-dimer and 
Table I. Laboratory findings of 214 patients with hepatic hemangioma.

\begin{tabular}{|c|c|}
\hline Parameters & Value \\
\hline Age, years & $55 \pm 15$ \\
\hline Sex, n (male/female) & $74 / 140$ \\
\hline \multicolumn{2}{|l|}{ Biochemistry } \\
\hline Total bilirubin, mg/dl & $0.6 \pm 0.3$ \\
\hline Albumin (g/dl) & $4.3 \pm 0.3$ \\
\hline ALT, U/1 & $20 \pm 14$ \\
\hline GGT, U/1 & $38 \pm 44$ \\
\hline ALP, U/1 & $232 \pm 78$ \\
\hline BUN, mg/dl & $14.5 \pm 3.9$ \\
\hline $\mathrm{Cr}, \mathrm{mg} / \mathrm{dl}$ & $0.69 \pm 0.19$ \\
\hline LDL-chol, mg/dl & $99 \pm 24$ \\
\hline Glucose, mg/dl & $101 \pm 29$ \\
\hline \multicolumn{2}{|l|}{ Hematology and coagulation } \\
\hline Hemoglobin, g/dl & $13.5 \pm 1.3$ \\
\hline $\mathrm{WBC}, / \mu 1$ & $5,500 \pm 1,600$ \\
\hline Platelet, $\times 10^{4} / \mathrm{mm}^{3}$ & $22.3 \pm 5.4$ \\
\hline PT, $\%$ & $94.3 \pm 13.1$ \\
\hline Fibrinogen, mg/dl & $275 \pm 71$ \\
\hline $\mathrm{TAT}^{\mathrm{a}}, \mathrm{ng} / \mathrm{ml}$ & $1.69 \pm 0.89$ \\
\hline D-dimer ${ }^{\mathrm{b}}, \mu \mathrm{g} / \mathrm{ml}$ & $1.03 \pm 0.72$ \\
\hline $\mathrm{FDP}^{\mathrm{c}}, \mu \mathrm{g} / \mathrm{ml}$ & $4.06 \pm 1.24$ \\
\hline \multicolumn{2}{|l|}{ Serology } \\
\hline M2BPGi, COI & $0.57 \pm 0.41$ \\
\hline AFP, ng/ml & $3.5 \pm 1.6$ \\
\hline PIVKA-II, mAU/ml & $19.1 \pm 6.6$ \\
\hline \multicolumn{2}{|l|}{ Associated liver diseases, n (\%) } \\
\hline Hepatitis B & $6(2.8)$ \\
\hline Hepatitis C & $5(2.3)$ \\
\hline Autoimmune hepatitis & $8(3.7)$ \\
\hline Primary biliary cholangitis & $2(0.9)$ \\
\hline Alcoholic liver disease & $6(2.8)$ \\
\hline Nonalcoholic steatohepatitis & $5(2.3)$ \\
\hline \multicolumn{2}{|l|}{ Concomitant diseases, n (\%) } \\
\hline Hypertension & $37(17.3)$ \\
\hline Hyperlipidemia & $21(9.8)$ \\
\hline Diabetes & $10(4.7)$ \\
\hline
\end{tabular}

The numbers of patients with detectable values were ${ }^{\mathrm{a}} \mathrm{n}=159,{ }^{\mathrm{b}} \mathrm{n}=137$ and ${ }^{c} n=27$. Values are presented as the mean \pm standard deviation or number $(\%)$.ALT, alanine transeferase; GGT, $\gamma$-glutamyltranspeptidase; ALP, alkaline phosphatase; BUN, blood urea nitrogen; Cr, creatinine; LDL, low density lipoprotein; WBC, white blood cell; PT, prothrombin time; TAT, thrombin-antithrombin III complex; FDP, fibrin and fibrinogen degradation products; M2BPGi, Mac-2 binding protein glycosylation isomer; AFP, $\alpha$-fetoprotein; PIVKA-II, protein induced by vitamin $\mathrm{K}$ absence-II.

FDP). The number of patients with the abnormal values of TAT, D-dimer, and FDP was significantly higher in the mixed group than in the homogeneous group (all $\mathrm{P}<0.0001$ ).
Table II. Ultrasonographic findings of 214 patients with hepatic hemangioma.

\begin{tabular}{lc}
\hline Characteristics & Value \\
\hline Echo pattern, $\mathrm{n}(\%)$ & \\
Hyperechoic & $159(74.3)$ \\
Hypoechoic & $2(0.9)$ \\
Isoechoic & $1(0.5)$ \\
Mixed & $52(24.3)$ \\
Size of hemangioma, $\mathrm{n}(\%)$ & \\
Small $(<20$ mm) & $145(67.8)$ \\
Medium $(20-40$ mm) & $48(22.4)$ \\
Large (>40 mm) & $21(9.8)$ \\
Location of hemangioma, $\mathrm{n}(\%)$ & \\
Right lobe & $180(84.1)$ \\
Left lobe & $32(15.0)$ \\
Bilateral & $2(0.9)$ \\
Number of hemangioma, $\mathrm{n}(\%)$ & \\
Single & $173(80.8)$ \\
Multiple & $41(19.2)$ \\
Portal vein diameter, mm & $10.8 \pm 2.0$ \\
Spleen index, mm ${ }^{2}$ & $1,295 \pm 558$ \\
\hline
\end{tabular}

Ninety-three patients were followed up for at least 12 months. The average follow-up period after the initial examination was 21.1 months (range, 12-29 months). There was no significant difference between the first and last data in the follow-up period in terms of hemangioma size, platelet count, fibrinogen level; and all of the TAT, D-dimer, and FDP values (data not shown).

Univariate and multivariate analyses were performed to assess for predictors of tumor size (Table VII). Albumin, $\mathrm{Cr}$, platelet count, fibrinogen level, PIVKA-II, portal vein diameter, and spleen index were significantly correlated with tumor size. To evaluate the significant factors contributing to the tumor size, albumin, Cr, platelet count, fibrinogen level, PIVKA-II, portal vein diameter, spleen index, the abnormal values of TAT, D-dimer and FDP were included in a stepwise multivariate analysis. TAT $(\mathrm{P}<0.05), \mathrm{D}$-dimer and FDP (both $\mathrm{P}<0.0001)$ were identified as independent factors related to the tumor size.

\section{Discussion}

Hepatic hemangiomas are the most common benign tumors of the liver, and have a prevalence of $0.4-7.3 \%$ on autopsy series and an incidence of $1.7 \%$ on abdominal US examination $(1,2)$. The prevalence in the present study was $1.3 \%$. The majority of the present patients were asymptomatic, and the lesions were detected incidentally during routine abdominal US examinations. Patients were asymptomatic even in the case of very large hemangiomas, although one patient who had a relatively large and superficially located hemangioma had abdominal distension.

We classified the internal echo patterns into homogeneous (homogeneous hyperechoic, homogeneous hypoechoic, 
Table III. Association between tumor size and clinical parameters in 214 patients with hepatic hemangioma.

\begin{tabular}{|c|c|c|c|c|}
\hline Parameters & Small $(n=145)$ & Medium (n=48) & Large $(n=21)$ & P-value \\
\hline Age, years & $53 \pm 15$ & $57 \pm 14$ & $59 \pm 14$ & 0.130 \\
\hline Male/female, $n$ & $47 / 98$ & $19 / 29$ & $8 / 13$ & 0.622 \\
\hline \multicolumn{5}{|l|}{ Biochemistry } \\
\hline Total bilirubin, mg/dl & $0.6 \pm 0.3$ & $0.6 \pm 0.3$ & $0.5 \pm 0.2$ & 0.693 \\
\hline Albumin, g/dl & $4.4 \pm 0.2$ & $4.4 \pm 0.3$ & $4.1 \pm 0.3^{\mathrm{a}, \mathrm{b}}$ & 0.001 \\
\hline ALT, U/1 & $21 \pm 15$ & $20 \pm 11$ & $18 \pm 9$ & 0.645 \\
\hline GGT, U/l & $39 \pm 46$ & $41 \pm 40$ & $30 \pm 32$ & 0.637 \\
\hline ALP, U/1 & $231 \pm 79$ & $232 \pm 76$ & $231 \pm 84$ & 0.997 \\
\hline BUN, mg/dl & $14.2 \pm 3.7$ & $14.6 \pm 4.3$ & $16.6 \pm 3.9^{c}$ & 0.038 \\
\hline $\mathrm{Cr}, \mathrm{mg} / \mathrm{dl}$ & $0.68 \pm 0.16$ & $0.71 \pm 0.24$ & $0.75 \pm 0.17$ & 0.248 \\
\hline LDL-chol, mg/dl & $98 \pm 24$ & $102 \pm 26$ & $99 \pm 20$ & 0.286 \\
\hline Glucose, mg/dl & $98 \pm 27$ & $104 \pm 34$ & $109 \pm 33$ & 0.147 \\
\hline \multicolumn{5}{|l|}{ Hematology } \\
\hline Hemoglobin, g/dl & $13.5 \pm 1.3$ & $13.7 \pm 1.2$ & $12.8 \pm 1.7^{\mathrm{b}, \mathrm{c}}$ & 0.029 \\
\hline $\mathrm{WBC}, / \mu 1$ & $5,600 \pm 1,600$ & $5,500 \pm 1,500$ & $5,400 \pm 1,600$ & 0.786 \\
\hline \multicolumn{5}{|l|}{ Serology } \\
\hline M2BPGi, COI & $0.55 \pm 0.42$ & $0.64 \pm 0.50$ & $0.77 \pm 0.40^{\mathrm{c}, \mathrm{d}}$ & 0.017 \\
\hline AFP, ng/ml & $3.4 \pm 1.5$ & $3.8 \pm 1.6$ & $3.3 \pm 2.0$ & 0.082 \\
\hline PIVKA-II, mAU/ml & $17.9 \pm 6.5$ & $21.0 \pm 6.4$ & $22.8 \pm 6.1^{\mathrm{a}, \mathrm{b}}$ & $<0.001$ \\
\hline Echo pattern, n (\%) & & & & $<0.001$ \\
\hline Homogeneous type & $144(99.3)$ & $18(37.5)$ & $0(0.0)$ & \\
\hline Mixed type & $1(0.7)$ & $30(62.5)$ & $21(100.0)$ & \\
\hline Portal vein diameter, $\mathrm{mm}$ & $10.4 \pm 1.9$ & $11.3 \pm 1.6^{\mathrm{c}}$ & $12.3 \pm 2.0^{\mathrm{a}}$ & $<0.001$ \\
\hline Spleen index, $\mathrm{mm}^{2}$ & $1,227 \pm 515$ & $1,368 \pm 527$ & $1,604 \pm 749^{c}$ & 0.042 \\
\hline
\end{tabular}

${ }^{\mathrm{a}} \mathrm{P}<0.01$ vs. small group; ${ }^{\mathrm{b}} \mathrm{P}<0.01$ vs. medium group; ${ }^{\mathrm{c}} \mathrm{P}<0.05$ vs. small group; ${ }^{\mathrm{d}} \mathrm{P}<0.05$ vs. medium group. Values are presented as the mean \pm standard deviation or number $(\%)$. ALT, alanine transeferase; GGT, $\gamma$-glutamyltranspeptidase; ALP, alkaline phosphatase; BUN, blood urea nitrogen; Cr, creatinine; LDL, low density lipoprotein; WBC, white blood cell; M2BPGi, Mac-2 binding protein glycosylation isomer; AFP, $\alpha$-fetoprotein; PIVKA-II, protein induced by vitamin $\mathrm{K}$ absence-II.

and isoechoic) and mixed (mixed hyperechoic and mixed hypoechoic) type (1). The sonographic appearance was homogeneous in $75.7 \%$ and mixed in $24.3 \%$ of the present lesions. Hemangiomas are usually visualized on US as well-defined, homogeneous, hyperechoic lesions. However, the appearance can vary in larger lesions because of intra-tumoral thrombosis, hemorrhage, fibrosis, or calcification, resulting in a heterogeneous or sometimes hypoechoic appearance $(7,8,10)$. In the present study, a mixed echo pattern was seen in 1 of 145 masses $(0.7 \%)$ with diameter $<20 \mathrm{~mm}$, in 30 of $48(62.5 \%)$ with diameter $20-40 \mathrm{~mm}$, and in all $21(100 \%)$ with diameter $>40 \mathrm{~mm}$. This result clearly demonstrates that the internal echo pattern becomes inhomogeneous with increasing size of the mass. Central degeneration resulting from thrombosis or hemorrhagic necrosis may cause the decreased echogenicity seen in part or completely throughout large hemangiomas (6). The size and internal echo pattern of hepatic hemangiomas may be caused by intra-tumoral thrombosis or hemorrhage. Some authors have reported that the heterogeneous appearance of larger lesions is the result of intra-tumoral thrombosis or hemorrhage (10) and that the internal echoes become increasingly inhomogeneous as the mass increases in size because of the histopathological presence of thrombosis, fibrosis, hemorrhage or calcification (1).

KMS is well known as a giant hepatic hemangioma characterized by a large vascular anomaly and consumption coagulopathy (11). The primary pathophysiology of KMS developing secondarily to hepatic hemangioma is considered as the entrapment of platelets in the vascular spaces of the hemangioma, and activation of coagulation and fibrinolysis mechanisms $(11,12)$. A proposed mechanism for the platelet trapping is adhesion to the endothelium of the hemangioma, with aggregation and activation of platelets. Excessive flow and shear stress within the vessels of the hemangioma can also contribute to platelet activation. Consumption of platelets and coagulation factors, as well as ongoing fibrinolysis, results in intralesional bleeding and enlargement of the hemangioma (13); consequently, hemangiomas may lead to KMS during a certain period of life (11). PT and activated partial thromboplastin time (APTT) are typically prolonged, fibrinogen is reduced, and FDP and D-dimer can be elevated. Localized intralesional bleeding may occur, thus enlarging the lesion and exacerbating the underlying process (14). 
Table IV. Association between echo pattern and clinical parameters in 214 patients with hepatic hemangioma.

\begin{tabular}{|c|c|c|c|}
\hline Parameters & Homogenous type $(n=162)$ & Mixed type $(n=52)$ & P-value \\
\hline Age, years & $54 \pm 15$ & $57 \pm 14$ & 0.153 \\
\hline Male/female, $\mathrm{n}$ & $54 / 108$ & $20 / 32$ & 0.611 \\
\hline \multicolumn{4}{|l|}{ Biochemistry } \\
\hline Total bilirubin, mg/dl & $0.6 \pm 0.3$ & $0.5 \pm 0.2$ & 0.628 \\
\hline Albumin, $\mathrm{g} / \mathrm{dl}$ & $4.4 \pm 0.2$ & $4.2 \pm 0.3$ & $<0.001$ \\
\hline ALT, U/1 & $20 \pm 14$ & $19 \pm 12$ & 0.973 \\
\hline GGT, U/1 & $38 \pm 45$ & $39 \pm 40$ & 0.903 \\
\hline ALP, U/1 & $234 \pm 82$ & $222 \pm 69$ & 0.364 \\
\hline BUN, mg/dl & $14.1 \pm 3.7$ & $15.7 \pm 4.4$ & 0.026 \\
\hline $\mathrm{Cr}, \mathrm{mg} / \mathrm{dl}$ & $0.68 \pm 0.16$ & $0.75 \pm 0.24$ & 0.046 \\
\hline LDL-chol, mg/dl & $100 \pm 24$ & $97 \pm 24$ & 0.539 \\
\hline Glucose, mg/dl & $97 \pm 26$ & $111 \pm 36$ & 0.014 \\
\hline \multicolumn{4}{|l|}{ Hematology } \\
\hline Hemoglobin, g/dl & $13.6 \pm 1.3$ & $13.2 \pm 1.4$ & 0.118 \\
\hline $\mathrm{WBC}, / \mu 1$ & $5,600 \pm 1,500$ & $5,500 \pm 1,700$ & 0.647 \\
\hline \multicolumn{4}{|l|}{ Serology } \\
\hline M2BPGi, COI & $0.55 \pm 0.40$ & $0.73 \pm 0.53$ & 0.074 \\
\hline $\mathrm{AFP}, \mathrm{ng} / \mathrm{ml}$ & $3.4 \pm 1.4$ & $3.8 \pm 1.9$ & 0.278 \\
\hline PIVKA-II, mAU/ml & $18.1 \pm 6.6$ & $22.1 \pm 5.7$ & $<0.001$ \\
\hline Tumor size, mm & $12.4 \pm 5.2$ & $40.3 \pm 18.8$ & $<0.001$ \\
\hline Portal vein diameter, $\mathrm{mm}$ & $10.5 \pm 1.9$ & $11.7 \pm 2.0$ & $<0.001$ \\
\hline Spleen index, $\mathrm{mm}^{2}$ & $1,240 \pm 529$ & $1,458 \pm 602$ & 0.018 \\
\hline
\end{tabular}

Values are presented as the mean \pm standard deviation. ALT, alanine transeferase; GGT, $\gamma$-glutamyltranspeptidase; ALP, alkaline phosphatase; BUN, blood urea nitrogen; Cr, creatinine; LDL, low density lipoprotein; WBC, white blood cell; M2BPGi, Mac-2 binding protein glycosylation isomer; AFP, $\alpha$-fetoprotein; PIVKA-II, protein induced by vitamin $\mathrm{K}$ absence-II.
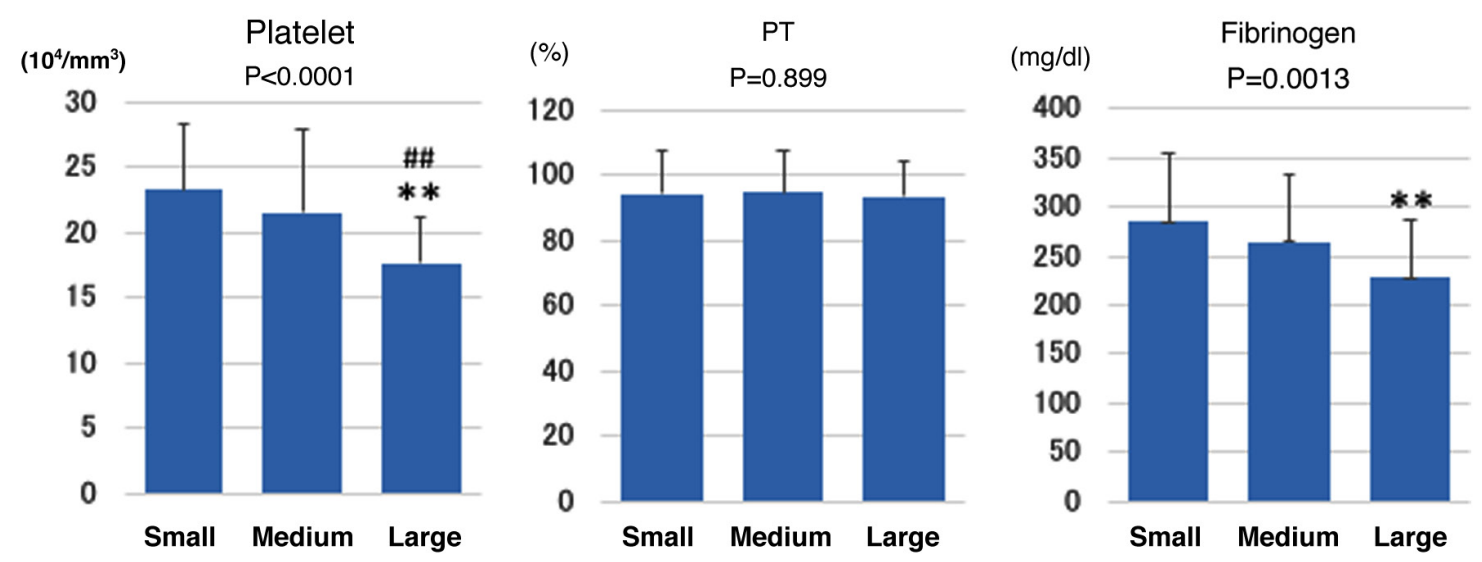

Figure 2. Association between tumor size and coagulation markers in 214 patients with hepatic hemangioma. P-values at the top indicate the comparison among the three groups by one way ANOVA. ${ }^{* *} \mathrm{P}<0.01$ vs. small group; ${ }^{\# \#} \mathrm{P}<0.01$ vs. medium group. $\mathrm{PT}$, prothrombin time.

Meanwhile hepatic hemangiomas are also a congenital vascular malformation, consisting of vascular channels ranging in size from capillary to cavernous and lined by a single layer of flattened endothelial cells within a thin fibrous stroma $(3,6,15)$. The pathogenesis of hemangioma is not well understood; however, these lesions can enlarge with time or can involute with regressive changes such as thrombosis, necrosis, calcification, and/or fibrosis (4). The internal echoes become inhomogeneous as the mass increases in size because of the histopathological presence of thrombosis, fibrosis, hemorrhage, or calcification (1). Bleeding disorders caused by excessive flow and shear stress within the vessels of the hemangioma result from intravascular coagulation within the tumor $(11,16)$. In the present study, patients with larger and mixed echo type hemangiomas had significantly lower platelet counts and fibrinogen levels than those of the other types, 
Table V. Association between tumor size and coagulation markers in 214 patients with hepatic hemangioma.

\begin{tabular}{|c|c|c|c|c|}
\hline Coagulation markers & Small $(n=145)$ & Medium (n=48) & Large $(n=21)$ & P-value \\
\hline \multicolumn{5}{|l|}{ TAT } \\
\hline Normal value & 145 & 46 & 12 & $<0.001$ \\
\hline Abnormal value & 0 & 2 & 9 & \\
\hline \multicolumn{5}{|l|}{ D-dimer } \\
\hline Normal value & 132 & 36 & 0 & $<0.001$ \\
\hline Abnormal value & 13 & 12 & 21 & \\
\hline \multicolumn{5}{|l|}{ FDP } \\
\hline Normal value & 145 & 48 & 11 & $<0.001$ \\
\hline Abnormal value & 0 & 0 & 10 & \\
\hline
\end{tabular}

TAT, thrombin-antithrombin III complex; FDP, fibrin and fibrinogen degradation products.

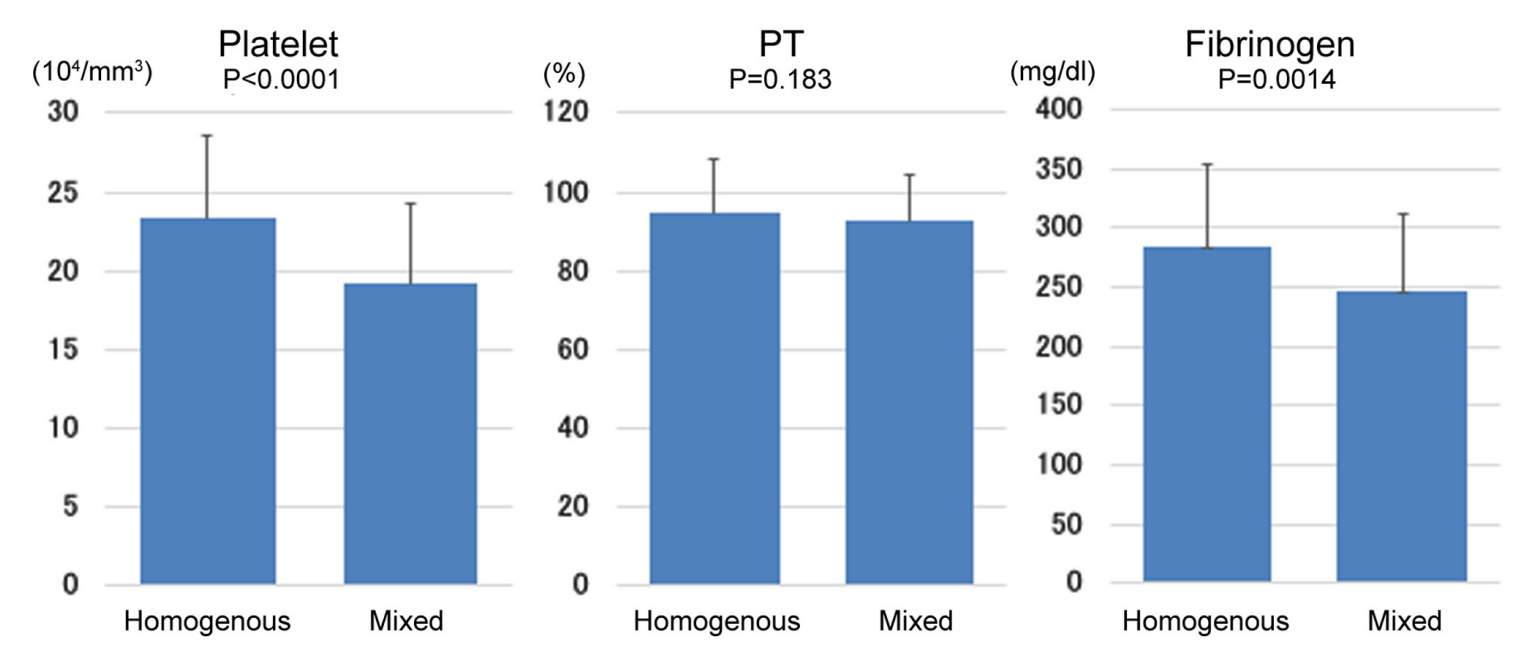

Figure 3. Association between echo pattern and coagulation markers in 214 patients with hepatic hemangioma. PT, prothrombin time.

and the values of TAT, D-dimer, and FDP were significantly elevated with increasing tumor size; those of the mixed echo group were significantly higher than those of the homogenous group. We examined independent factors among the clinical parameters for the size of hemangiomas and found that serum TAT, D-dimer and FDP were important independent factors. From these results, we speculate that lesion size and echogenicity on US are associated with coagulation factors, especially TAT, D-dimer, and FDP. These factors are well known as hemostatic and fibrinolytic biomarkers that are considered useful for the diagnosis of thrombosis $(17,18)$. Therefore, we can conclude that differences in the size and echogenicity of hemangiomas are caused by intra-tumoral thrombosis and subsequent hemorrhage.

TAT is induced by thrombin and is direct evidence of the increased activation of coagulation and the consumption of inhibitors. Therefore, the TAT level reflects the early phase of thrombosis and is a useful predictor of prethrombotic state. Elevated TAT reflects activation of the coagulation system and indicates a hypercoagulable state in which, together with the stasis of blood flow that occurs within the vessels of a hemangioma, thrombosis can easily form (18). D-dimer, a product of fibrin degradation, reflects both the activation of fibrinolysis and the severity of the hypercoagulable state. Elevated D-dimer can result from the marked activation of blood coagulation, leading to massive formation of intravascular thrombosis. Accordingly, increased plasma levels of D-dimer are considered to reflect the presence of intravascular thrombosis (19). FDP consists of both fibrin and fibrinogen-degradation products, and the levels of FDP are often closely correlated with D-dimer. More than $50 \%$ of patients with FDP values $>5.0 \mu \mathrm{g} / \mathrm{ml}$ have some thrombosis, suggesting that these patients may have a hypercoagulable state. Thus, high plasma levels of FDP, including D-dimer, reflect a thrombotic state and suggest the presence of venous thromboembolism (17). As D-dimer and FDP reflect secondary fibrinolysis after clot formation, they may not be useful as early predictive markers for thromboembolism. In contrast, the TAT level is direct evidence of the increased activation of coagulation and the consumption of inhibitors. The half-life of TAT is short, whereas that of D-dimer is long. Thus, TAT levels increase rapidly and diminish relatively soon after the onset of thromboembolism (18). In the present study, almost all patients with prominent elevation of D-dimer and FDP also had high TAT levels, suggesting a hypercoagulable state and subsequent repeated formation of thromboembolism and fibrinolysis within the vessels of hemangiomas. 
Table VI. Association between echo pattern and coagulation markers in 214 patients with hepatic hemangioma.

\begin{tabular}{lccc}
\hline Coagulation markers & Homogenous $(\mathrm{n}=162)$ & Mixed $(\mathrm{n}=52)$ & P-value \\
\hline TAT & 162 & 40 & $<0.001$ \\
Normal value & 0 & 12 & $<0.001$ \\
Abnormal value & 148 & 19 & $<0.001$ \\
D-dimer & 14 & 33 & \\
$\quad$ Normal value & 162 & 42 & 10 \\
Abnormal value & 0 & & \\
FDP & & 10 & \\
Normal value & Abnormal value & & \\
\hline
\end{tabular}

TAT, thrombin-antithrombin III complex; FDP, fibrin and fibrinogen degradation products.

Table VII. Univariate and multivariate regression analyses of factors associated with tumor size in 214 patients with hepatic hemangioma.

\begin{tabular}{|c|c|c|c|c|c|}
\hline \multirow[b]{2}{*}{ Variables } & \multicolumn{2}{|c|}{ Univariate analysis } & \multicolumn{3}{|c|}{ Multivariate analysis } \\
\hline & $\mathrm{r}$ & P-value & $\beta$ & $\mathrm{SE}(\beta)$ & P-value \\
\hline Albumin & -0.162 & 0.018 & 2.680 & 3.04 & 0.379 \\
\hline $\mathrm{Cr}$ & 0.156 & 0.023 & 0.625 & 3.97 & 0.875 \\
\hline Platelet & -0.305 & 0.001 & -0.237 & 0.14 & 0.094 \\
\hline Fibrinogen & 0.223 & 0.001 & -0.020 & 0.01 & 0.052 \\
\hline TAT & a & & 9.419 & 3.67 & 0.011 \\
\hline D-dimer & a & & 11.959 & 2.02 & $<0.001$ \\
\hline FDP & a & & 30.690 & 4.10 & $<0.001$ \\
\hline PIVKA-II & 0.263 & $<0.001$ & 0.203 & 0.11 & 0.076 \\
\hline Portal vein diameter & 0.329 & $<0.001$ & 0.735 & 0.39 & 0.064 \\
\hline Spleen index & 0.181 & 0.008 & -0.0001 & $<0.01$ & 0.961 \\
\hline
\end{tabular}

${ }^{a}$ TAT, D-dimer and FDP were analyzed using a $\chi^{2}$ test (see Table V). Cr, creatinine; TAT, thrombin-antithrombin III complex; FDP, fibrin and fibrinogen degradation products; PIVKA-II, protein induced by vitamin $\mathrm{K}$ absence-II.

Fibrinogen is converted to fibrin by activated thrombin and is a key protein in the coagulation pathway, clot formation, and in supporting platelet aggregation, which forms the final step of the coagulation cascade. Platelets are also crucial in the coagulation system (20). A previous study reported that plasma fibrinogen levels were correlated with platelet counts (21). Our results demonstrated that patients with larger lesions or mixed echo pattern had significantly lower platelet counts and fibrinogen levels. We speculated that there could be two possible causes for the decrease in fibrinogen levels and platelet counts as complications associated with hemangiomas, which are, respectively: (1) enhanced thrombotic formation through platelet aggregation, (2) increased consumption of fibrinogen caused by increased activation of fibrinolysis; and (1) entrapment of platelets in the vascular spaces of the hemangioma and activation of coagulation, (2) consumption of platelets in addition to ongoing fibrinolysis. In several previous studies of 51Cr-platelets or 125I-fibrinogen (22-25), there was a rapid removal of radioactive platelets and fibrinogen from the circulation in hemangioma patients. Low platelet counts and fibrinogen levels were considered to be the result of intravascular coagulation in the hemangioma, and it was concluded that platelets and fibrinogen were consumed or degraded in the hemangioma. These decreases in platelet counts and fibrinogen levels were not low enough to be directly related to systemic bleeding disorders, but rather an indirect effect of intra-tumoral hemorrhage, along with thrombosis.

Portal vein diameter and splenic index were significantly elevated in our patients with larger tumor size and mixed echo pattern. An experimental model with portal hypertension has shown that portal pressure positively correlated with spleen size (26). Portal hypertension develops because of increased intrahepatic vascular resistance, often caused by chronic liver disease, that leads to structural distortion by such as fibrosis and microvascular thrombosis $(27,28)$. In our study, M2BPGi (29,30), a biochemical marker of liver fibrosis, was 
significantly elevated in patients with relatively large hemangiomas; in addition, chronic liver disease as underlying disease was present in 15 of 145 patients $(10.3 \%)$ in the small group, in 11 of $48(22.9 \%)$ in the medium group, and in 6 of $21(28.6 \%)$ in the large group, with significant difference in each group $(\mathrm{P}<0.05$; data not shown). This finding suggests that the values of M2BPGi in each group might be influenced by the presence of chronic liver disease as underlying disease. However; the mean M2BPGi values in the large group were within normal values and were $<1.00 \mathrm{COI}$, which is the optimal cutoff value in patients with fibrosis grade F1 (30). Therefore the elevation of portal vein diameter and spleen index seen in our study might be not caused by liver fibrosis. Based on our results and those of previous studies, we speculate that these high values may have been caused by increased vascular resistance due to microvascular thrombosis within hemangiomas $(27,28)$, regional circulatory disorders and liver fibrosis surrounding the hemangiomas $(31,32)$, and possibly by intrahepatic fibrosis due to chronic liver disease. Although the effects of these factors on intrahepatic vascular resistance leading to portal hypertension are not certain, it is possible that the existence of hepatic hemangiomas may play a role in triggering portal hypertension.

The significance of change in size of hemangiomas is controversial. Several studies that have examined the natural course of hepatic hemangiomas reported that the vast majority remained stable in size $(3,5,6,33)$. Among the studies that have reported hemangioma enlargement, there is general agreement that these lesions increase in size following intra-tumoral thrombosis or hemorrhage $(2,34)$. In contrast, some authors have reported that hemangiomas might undergo degeneration and fibrous replacement, which would explain the size decrease in one of the hemangiomas (35), and others have reported that the size decrease in hemangiomas is associated with the changes in hepatic blood flow and intrahepatic environment that occur with the progression of liver fibrosis $(3,31,32)$, with regional circulatory disorders in the peripheral liver that are triggered by intra-tumoral thrombus formation (36), and with suppression caused by pressure from the surrounding tissue after tumor size has increased to a certain degree (37).

In the present study, most of the hepatic hemangiomas remained stable in size and there was little change in echo pattern during their natural course. The average follow-up period after the initial examination was 21.1 months (range, 12-29 months) and the number of follow-up patients was 93 . Our study suffered from limitations regarding the length of follow up and the number of patients; therefore, we could not reach significant conclusions from our data. A longer follow-up period and a larger number of patients are needed to solve this issue. Because tumors $<20 \mathrm{~mm}$ in diameter accounted for more than half of the hemangiomas in the present study, measurement error could easily have occurred in these patients during the follow-up period. It is necessary to find an appropriate method to reduce the possibility of false positive findings caused by such error. However, based on our results and those of previous studies, we can speculate that the change in the size of hemangiomas depends on the various influences of growth factors caused by intra-tumoral thrombosis, hemorrhage, and necrosis; inhibitory factors caused by intra-tumoral degeneration and fibrous replacement; or extra-tumoral circulatory disorders, liver fibrosis, or pressure from surrounding tumor.
In conclusion, our study is the first to demonstrate a relationship between tumor size and the internal echo pattern of hepatic hemangiomas and coagulation factors. Although further studies are required to evaluate the exact relationship, we speculate that differences in tumor size and echogenicity are caused by intra-tumoral thrombosis and subsequent hemorrhage.

\section{Acknowledgements}

Not applicable.

\section{Funding}

No funding was received.

\section{Availability of data and materials}

The datasets used and/or analyzed during the current study are available from the corresponding author on reasonable request.

\section{Authors' contributions}

SM was involved in conceptualization, methodology, formal analysis, investigation, data curation, the confirmation of data authenticity, review and editing of the manuscript, visualization, supervision and project administration. MK was involved in conceptualization, methodology, formal analysis, statistical analysis, data curation, the confirmation of data authenticity, review and editing of the manuscript, visualization and supervision. TM performed data curation. HI was involved in the acquisition of data and confirmation of data authenticity, review and editing of the manuscript, visualization and supervision. All authors read and approved the final manuscript.

\section{Ethics approval and consent to participate}

The present study was approved by our hospital ethical committee (Hino Hospital, Tottori, Japan; approval no. 2018-4) and performed according to the Declaration of Helsinki. Consent to participate was acquired using an opt-out system.

\section{Patient consent for publication}

Consent for publication was acquired using an opt-out system.

\section{Competing interests}

The authors declare that they have no competing interests.

\section{References}

1. Ito $\mathrm{H}$, Tsujimoto $\mathrm{F}$, Nakajima $\mathrm{Y}$, Igarashi $\mathrm{G}$, Okamura $\mathrm{T}$, Sakurai M, Nobuoka S and Otsubo T: Sonographic characterization of 271 hepatic hemangiomas with typical appearance on CT imaging. J Med Ultrasonics 39: 61-68, 2012.

2. Li J, Huang L, Liu C, Yan J, Xu F, Wu M and Yan Y: New recognition of the natural history and growth pattern of hepatic hemangioma in adults. Hepatol Res 46: 727-733, 2016

3. Okano H, Shiraki K, Inoue H, Ito T, Yamanaka T, Deguchi M, Sugimoto K, Sakai T, Ohmori S, Murata K, et al: Natural course of cavernous hepatic hemangioma. Oncol Rep 8: 411-414, 2001 
4. Kim GE, Thung SN, Tsuji WM and Ferrell LD: Hepatic cavernous hemangioma: Underrecognized associated histologic features. Liver Int 26: 334-338, 2006.

5. Gandolfi L, Leo P, Solmi L, Vitelli E, Verros G and Colecchia A Natural history of hepatic hemangiomas: Clinical and ultrasound study. Gut 32: 677-680, 1991.

6. Gibney RG, Hendin AP and Cooperberg PL: Sonographically detected hepatic hemangiomas: Absence of change over time. Am J Rentgenol 149: 953-957, 1967.

7. Erdogan D, Busch ORC, Delden OM, Bennink RJ, Kate FJ, Gouma DJ and Guiik TM: Management of liver hemangiomas according to size and symptoms. J Gastroenterol Hepatol 22: 1953-1958, 2007.

8. Duxburg MS and Garden OJ: Giant hemangioma of the liver: Observation or resection? Dig Surg 27: 7-11, 2010.

9. Carlo ID, Koshy R, Mudares SA and Ardiri A: Giant carvenous liver hemangiomas: Is it the time to change the size categories? Hepatobiliary Pancreat Dis Int 15: 21-29, 2016.

10. HermanP,CoataML,MachadoMA,Pugliese V,D'Albuquerque LA Machado MC, Gama-Rodrigues JJ and Saad WA: Management of hepatic hemangiomas: A 14-year experience. J Gastrointest Surg 9: 853-859, 2005 .

11. Bozkaya H, Cinar C, Unalp OV, Parildar M and Oran I: Unusual treatment of Kasabach-Merritt syndrome secondary to hepatic hemangioma: Embolization with bleomycin. Wien Klin Wochenschr 127: 488-490, 2015.

12. Hall GW: Kasabach-Merritt syndrome: Pathogenesis and management. Br J Hematol 112: 851-862, 2001.

13. Rodriguez V, Lee A, Witman PM and Anderson PA: Kasabachmerritt phenomenon: Case series and retrospective review of the mayo clinic experience. J Pediatr Hematol Oncol 31: 522-526, 2009.

14. O'Rafferty C, O'Regan GM, Irvine AD and Smith OP: Recent advances in the pathobiology and management of Kasabach-Merritt phenomenon. Br J Hematol 171: 38-51, 2015.

15. Hoekstra LT, Bieze M, Erdogan D, Roelofs JJ, Beuers UH and Gulik TM: Management of giant hemagiomas: An update. Expert Rev Gastroenterol Hepatol 7: 263-268, 2013.

16. Mewes T, Moldenhauer H, Pfeifer J and Papenberg J: The Kasabach-Merritt syndrome: Severe bleeding disorder caused by celiac arteriography-reversal by heparin treatment. Am J Gastroenterl 84: 965-971, 1989

17. Wada $\mathrm{H}$ and Sakuragawa $\mathrm{N}$ : Are fibrin-related markers useful for the diagnosis of thrombosis? Semin Thromb Hemost 34: 33-38, 2008.

18. Lee SY, Niikura T, Iwakura T, Sakai Y, Kuroda R and Kurosaka M: Thrombin-antithrombin III complex tests: A usefu screening tool for postoperative venous thromembolism in lower limb and pelvic fracture. J Orthop Surg 25: 1-6, 2017.

19. Deng Y, He L, Yang J and Wang J: Serum D-dimer as an indicator of immediate mortality in patients with in-hospital cardiac arrest. Thromb Res 143: 161-165, 2016.

20. Tang L, Liu K, Wang J, Wang C, Zhao P and Liu J: High preoperative plasma fibrinogen levels are associated with distant metastases and impaired prognosis after curative resection in patients with colorectal cancer. J Surg Oncol 102: 428-432, 2010.

21. Wang GY, Jiang N, Yi HM, Wang GS, Zhang JW, Li H, Zhang J, Zhang Q, Yang Y and Chen GH: Pretransplant elevated plasma fibrinogen level is a novel prognostic predictor for hepatocellular carcinoma recurrence and patient survival following liver transplantation. Ann Transplant 21: 125-130, 2016.

22. Suzuki H, Nimura Y, Kamiya J, Kondo S, Nagino M, Kanai M and Miyachi M: Preoperative transcatheter arterial embolization for giant carvenous hemangioma of the liver with consumption coagulopathy. Am J Gastroenterol 92: 688-691, 1997.
23. Blix S and Aas K: Giant hemangioma, thrombocytopenia, fibrinogenopenia, and fibrinolytic activity. Acta Med Scand 169: 63-70, 1961.

24. Wochner RD, Kulapongs P and Bachmann F: 125I-fibrinogen turnover and coagulation studies in a patient with KasabachMerritt syndrome. J Lab Clin Med 70: 997, 1967.

25. Hillman RS and Philips LL: Clotting-fibrinolysis in a cavernous hemangioma. Am J Dis Child 113: 649-653, 1967.

26. Iwaki Y: Pathophysiology of portal hypertension. Cli Liver Dis 18: 281-291, 2014.

27. McConnell $\mathrm{M}$ and Iwakiri Y: Biology of portal hypertension. Hepatol Int 12 (Suppl 1): S11-S23, 2018.

28. Mehta G, Gustot T, Mookerjee RP, Garcia-Pagan JC, Fallon MB, Shah VH, Moreau R and Jalan R: Inflammation and portal hypertension-The undiscovered country. J Hepatol 61: 155-163, 2014.

29. Shirabe K, Bekki Y, Gantumur D, Araki K, Ishii N, Kuno A, Narimatsu $\mathrm{H}$ and Mizokami M: Mac-2 binding protein glycan isomer (M2BPGi) is a new serum biomarker for assessing liver fibrosis: More than a biomarker of liver fibrosis. J Gastroenterol 53: 819-826, 2018.

30. Toshima T, Shirabe K, Ikegami T, Yoshizumi T, Kuno A, Togayachi A, Gotoh M, Narimatsu H, Korenaga M, Mizokami M, et al: A novel serum marker, glycosylated Wisteria floribunda agglutinin-positive Mac-2 binding protein (WFA(+)-M2BP), for assessing liver fibrosis. J Gastroenterol 50: 76-84, 2015.

31. Kobayashi T, Kawano M, Tomita Y, Tamano M, Saigusa S, Horinaka M, Monma T, Koguma T, Yanagisawa N, Ohe T, et al: Follow-up study of hepatic hemangiomas. Nippon Shokakibyo Gakkai Zasshi 92: 41-46, 1995 (In Japanese).

32. Miyaki D, Aikata H, Waki K, Murakami H, Hashimoto Y, Nagaoki H, Katamura Y, Kataoka T, Takagi S, Hiramatsu K, et al: Significant regression of a cavernous hepatic hemangioma to a sclerosed hemangioma over 12 years: A case study. Nippon Shokakibyo Gakkai Zasshi 108: 954-961, 2011 (In Japanese).

33. Yeh WC, Yang PM, Huang GT, Sheu JC and Chen DS Long-term follow-up of hepatic hemangiomas by ultrasonography: With emphasis on the growth rate of the tumor. Hepatogastroenterology 54: 475-479, 2007.

34. Nghiem HV, Bogost GA, Ryan JA, Lund P, Freeny PC and Rice KM: Cavernous hemangiomas of liver: Enlargement over time. Am J Rentgenol 169: 137-140, 1997.

35. Bree RL, Schwab RE and Neiman HL: Solitary echogenic spot in the liver: Is it diagnostic of a hemangioma? Am J Rentgenol 140: 41-45, 1983.

36. Tsumaki N, Waguri N, Yonayama O, Hama H, Kawahisa J, Yokoo K, Aiba T, Furukawa K, Sugimura K, Igarashi K, et al: A case of sclerosed hemangioma with a significant morphological change over a period of 17 years. Kanzo 49: 268-274, 2008.

37. Ogawa K, Takeuchi K, Okuda C, Tamura T, Koizumi Y, Koyama R, Imamura T, Inoue Y and Arase Y: Change in size of hepatic hemangiomas during long-term observation: 80 lesions with observation for over 10 years. Jpn J Med Ultrasonics 41: 749-756, 2014.

This work is licensed under a Creative Commons Attribution-NonCommercial-NoDerivatives 4.0 International (CC BY-NC-ND 4.0) License. 